\title{
T3 subclassification using the EMD/mesorectum ratio predicts neoadjuvant chemoradiation outcome in T3 rectal cancer patients
}

\author{
1,2 LIJUN SHEN, MD, 2,3 YIQUN SUN, MD, 1,2 HUI ZHANG, PhD, 1,2 JING ZHANG, MD, 1,2 WEIJUAN DENG, MD, \\ ${ }_{1,2}^{1,2}$ YQI WANG, MD, ${ }^{1,2}$ YE YAO, PhD, ${ }^{1,2}$ LIFENG YANG, MD, ${ }^{1,2} \mathrm{JI} Z \mathrm{ZHU}, \mathrm{MD},{ }^{2,3}$ TONG TONG, MD, ${ }^{1,2}$ LIPING LIANG, PhD and \\ 1,2 ZHEN ZHANG, MD
}

'Department of Radiation Oncology, Fudan University Shanghai Cancer Center, Shanghai, China

${ }^{2}$ Department of Oncology, Shanghai Medical College, Fudan University, Shanghai, China

${ }^{3}$ Department of Radiology, Fudan University Shanghai Cancer Center, Shanghai, China

Address correspondence to:

Zhen Zhang

E-mail: zhen_zhang@fudan.edu.cn

Liping Liang

E-mail: Ilp_8521@hotmail.com

Lijun Shen and Yiqun Sun have contributed equally to this work and share co-first authorship.

Objective: To evaluate the feasibility of the EMD (extramural depth)/mesorectum ratio as a marker for T3 rectal cancer and its ability to predict tumour response to neoadjuvant chemoradiation and survival.

Methods: From 2010 to 2016, 284 T3 rectal cancer patients who underwent high resolution MRI before neoadjuvant chemoradiation were enrolled. The EMD was defined as the distance from the outer edge of the muscularis propria to the outermost edge of the tumour. The measurement of the tumour EMD and mesorectum was in the same layer and their ratio was calculated. Receiver operating characteristic analysis and relative area under the curve statistics were used to choose the cut-off value. The association of the EMD/mesorectum ratio and other MRI or clinical factors with the tumour regression grade (TRG) was analysed. Cox regression analysis was used to estimate independent risk factors for disease-free survival (DFS) and overall survival (OS). Results: The mean EMD/mesorectum ratio was $0.47 \pm 0.3$. We chose an $\mathrm{EMD} /$ mesorectum ratio of 0.5 in further analyses after receiver operating characteristic analysis. Of 284 patients, 177 (62.3\%) had an EMD/mesorectum ratio $\leq 0.5$. Patients with an $\mathrm{EMD} /$ mesorectum ratio $\leq 0.5$ had a higher TRG $0-1$ rate than patients with a ratio $>0.5$ ( $53.1 \%$ vs $36.4 \%$, $p=0.006$ ). A multivariate analysis identified that an $\mathrm{EMD} /$ mesorectum ratio >0.5 [hazard ratio (HR) 2.020; $p=0.028]$ and ypTNM II-III (HR 3.550; $p=0.017$ ) were independent prognostic factors to indicate decreased DFS. For OS, only patients with TRG 2-3 had decreased OS compared with patients with TRG O-1 (HR 2.959; $p=0.035$ ).

Conclusion: When the EMD/mesorectum ratio was applied to categorize $T 3$ rectal cancer patients, the ratio of 0.5 can be used as a cut-off value for $\mathrm{T} 3$ rectal cancer. Patients with a ratio $\leq 0.5$ had a higher response rate and better DFS. However, further validation is needed in a larger sample of patients.

Advances in knowledge: The $\mathrm{EMD} /$ mesorectum ratio may serve to predict tumour response to neoadjuvant chemoradiation and survival in T3 rectal cancer patients.

\section{INTRODUCTION}

For more than 10 years, neoadjuvant chemoradiation has been established as the standard of care in patients with locally advanced rectal cancer. ${ }^{1-3}$ The current trends in treatment have shifted toward a more personalized option. There is an increasing need for pre-operative imaging methods to non-invasively select high-risk patients to receive intensified local or systemic treatment, whereas low-risk patients may decrease their treatment intensity to achieve a better quality of life.

Stage T3 rectal cancer is the most heterogeneous patient subgroup due to the variation of mesorectum thickness. ${ }^{4}$ Whether all $\mathrm{T} 3$ rectal cancer patients require chemoradiation is controversial. ${ }^{5}$ Some studies have reported the prognostic influence of the mesorectal infiltration depth in pathology., 
Since chemoradiation will lead to tumour downstaging and fibrosis for most cancers, a precise measurement of the pre-operative depth of extramural tumour spread could provide accurate prognostication.

The MERCURY study has demonstrated that MR imaging is feasible and reproducible in a multicentre setting and yields data equivalent to histopathological results regarding the preoperative prediction of the depth of extramural tumour spread. ${ }^{8}$ Moreover, the extramural depth (EMD) has been shown to be an independent prognostic factor by MR. ${ }^{9}$

According to the European Society for Medical Oncology guidelines, T3 disease is further classified into T3a $(<1 \mathrm{~mm}), \mathrm{T} 3 \mathrm{~b}$ (1-5 mm), T3c (5-15 mm) and T3d (> $15 \mathrm{~mm})$ based on the depth of invasion beyond the muscularis propria. ${ }^{10}$ Although the subclassification is not validated in any randomized trials and have not been incorporated in any of the TNM versions, they may provide more personalized options for staging T3 rectal cancer. However, there may be limitations for the classification due to the variation of mesorectum thickness. The thickness of the mesorectal fat may differ due to body figure, tumour location and direction. Within the Chinese population, a thickness of mesorectum $<15 \mathrm{~mm}$ is found in most positions and in the majority of patients which leads to a lower T3d proportion. ${ }^{11}$ In clinical practice, T3a with depth of invasion less than $1 \mathrm{~mm}$ is also difficult to measure. Therefore, adding the EMD/mesorectum ratio to $\mathrm{T} 3$ subclassification would be a better evaluation choice considering these situations in $\mathrm{T} 3$ patients.

This study aimed to evaluate the feasibility of the EMD/ mesorectum ratio as part of the $\mathrm{T} 3$ subclassification and its ability to predict tumour response to neoadjuvant chemoradiation and survival in T3 rectal cancer patients.

\section{METHODS AND MATERIALS}

\section{Patients}

A consecutive cohort of 284 patients with MRI-staged locally advanced (cT3 and N0-2) rectal cancer who were treated with neoadjuvant chemoradiotherapy followed by surgery at Fudan University Shanghai Cancer Center between 2010 and 2016 was identified. All of their medical records were retrospectively reviewed. All of the patients were evaluated by MRI and underwent conventional radiation with concurrent 5-fluorouracil-based chemotherapy. The mean radiation dose was $50 \mathrm{~Gy}$ (range 45-55 Gy) with a daily fraction of 1.8-2.0 Gy using a 6 $\mathrm{mV}$ linear accelerator. Radiation treatments were performed according to institutional protocols. Surgery was planned for 6-10 weeks after the pre-operative treatments, and a total mesorectal excision procedure was recommended.

Adjuvant chemotherapy regimens, including FOLFOX, capecitabine \pm oxaliplatin or 5-FU, were decided by each physician. Approximately 6 months of total perioperative treatment is recommended. The protocol was approved by the hospital's Medical Ethics Committee.

\section{TRG evaluation}

The 4-point TRG (tumour regression grade) system graded on a scale of 0 to 3, which was recommended by the AJCC Cancer
Staging Manual, seventh Edition and the College of American Pathologists Guidelines as modified from Ryan et al. ${ }^{12,13}$ In this system, TRG 0 was for complete response (no viable cancer cells), TRG 1 for moderate response (only small clusters or single cancer cells remaining), TRG 2 for minimal response (residual cancer remaining, but with predominant fibrosis) and TRG 3 for poor response; minimal or no tumour killed, extensive residual cancer). Patients with TRG0-1 had good response while TRG2-3 had poor response.

\section{MR imaging and evaluation}

The primary staging MRI was performed before CRT. Patients were imaged in a 3.0 Tesla (T) MRI (Signa Horizon, GE Medical Systems, Milwaukee, WI) using a phased-array body coil. The standard imaging protocol consisted of a sagittal T2 weighted (T2W) fast spin echo and oblique axial thin-section T2W, which were used for measuring the maximal EMD. Patients did not receive bowel preparation, antispasmodic medication or rectal distention before the MR examinations. Two gastrointestinal radiologists, who were blinded to the information obtained at surgery and pathological analysis, reviewed the imaging set.

For each tumour, the maximal EMD of spread from the outer edge of the low-signal intensity longitudinal muscularis propria to the outermost edge of the tumour was measured and recorded using workstation calipers. The distance of the mesorectum was defined as the distance of outer edge of the muscularis propria of the rectum to the mesorectal fascia (MRF). The measurement of the tumour EMD and mesorectum occurred in the same layer. The final value of the EMD and the distance of the mesorectum were calculated by the mean of the 2 measurements. The definition of stage T3a-T3d tumours was taken from the European Society for Medical Oncology criteria based on the EMD beyond the muscularis propria (T3a: $<1 \mathrm{~mm}, \mathrm{~T} 3 \mathrm{~b}: 1-5 \mathrm{~mm}, \mathrm{~T} 3 \mathrm{c}: 5-15$ $\mathrm{mm}$, T3d: $>15 \mathrm{~mm}) .{ }^{10}$ The $\mathrm{cN}$ stage $(\mathrm{cN}-, \mathrm{N}+)$ was retrieved from the MRI at primary staging according to the size criteria $(>3 \mathrm{~mm})$ and the irregular margins, T2 and enhancement heterogeneity. A primary tumour located less than $5 \mathrm{~cm}$ from the anal margin was classified as a low rectal cancer. The association to the MRF was also evaluated. A measured distance of $1 \mathrm{~mm}$ or less on the thin-section T2W images was indicative of MRF involvement. Extramural vascular invasion (EMVI) was considered positive if certain morphological features were seen on $3 \mathrm{~mm}$ slices: a serpiginous extension of tumour signal within a vascular structure leading to vessel expansion or irregular contouring of the vessel border (vessel was seen as a tubular structure containing signal void on T2W images shown in continuity on adjacent slices). ${ }^{14,15}$

\section{Statistical analysis}

Continuous variables were expressed as the means and SD with range, and the categorical variables were expressed as the frequency and percentage. Receiver operating characteristic (ROC) analysis and relative area under the curve statistics were used, and the ratio closest to the point with the maximum sensitivity and specificity was selected as the optimal cut-off value of EMD/mesorectum ratio. The association of the EMD/ mesorectum ratio and other MRI or clinical factors with the 
TRG score was analysed by the $\mathrm{X}^{2}$ test and Fisher's exact test. Survival curves were generated using the Kaplan-Meier method, and groups were compared using the log-rank test. Univariate and multivariate analyses to identify prognostic predictors were performed using the Cox proportional hazard model. Variables with $p<0.05$ for univariate analysis were entered into the multivariate analyses. The intraclass correlation coefficient (ICC) was calculated to assess the interobserver reliability of EMD, the distance of the mesorectum and the $\mathrm{N}$ stage.

Statistical analysis was performed using the SPSS statistical software package, v. 18.0 (SPSS Inc., Chicago, IL). A two-sided $p<0.05$ was considered to be statistically significant.

\section{RESULTS}

\section{Patient characteristics}

A total of 284 MRI-staged T3 rectal cancer patients were analysed in this study. The study population was predominantly male $(71.5 \%)$ with a mean age of 55 years (range $22-80$ years). After re-evaluation of extramural invasion depth, the number of patients with T3a, T3b, T3c and T3d was 3 (1.1\%), 184 (64.8\%), $91(32.0 \%)$ and $6(2.1 \%)$ before chemoradiation, respectively. The mean EMD was $4.9 \pm 3.3 \mathrm{~mm}$, and the mean distance of the mesorectum was $13.1 \pm 6.9 \mathrm{~mm}$. The number of patients with N0, N1 and N2 on their MRI was 20 (7.0\%), 119 (41.9\%) and 145 (51.1), respectively. The interobserver reliability revealed good agreement for the EMD measurement (ICC $=0.892)$, distance of the mesorectum $(\mathrm{ICC}=0.843)$ and $\mathrm{N}$ stage $(\mathrm{ICC}=0.850)$. Of these patients, $133(46.8 \%)$ patients had a good tumour response with TRG $0-1$, and 60 (21.1\%) patients achieved pCR. Only one patient had a positive circumferential resection margin (CRM), therefore CRM was not included in further analyses. The median follow-up for survivors was 20.2 months (range 1-79.6 months). $12(4.2 \%)$ patients developed local recurrence, 34 (12.0\%) patients developed distant metastasis and 12 (4.2\%) patients had died by the time of last follow-up. The 5 -year disease-free survival (DFS) and overall survival (OS) were 68.1 and $80.8 \%$, respectively.

\section{Correlation between the EMD/mesorectum ratio and patient characteristics}

The mean EMD/mesorectum ratio was $0.47 \pm 0.3$. In the ROC analysis, the area under the curve of EMD/mesorectum ratio was $0.606(p=0.025)$ for DFS, $0.557(p=0.386)$ for OS and 0.535 $(p=0.407)$ for $\mathrm{pCR}$. As the value only had significant difference in predicting DFS in ROC analysis, the optimal EMD/mesorectum ratio of 0.52 in it was defined the cut-off value. We chose an EMD/ mesorectum ratio of 0.5 for further analyses because of feasibility in the clinical practice. Figure 1 demonstrated an example with EMD/mesorectum ratio $\leq 0.5$ while Figure 2 with EMD/ mesorectum ratio $>0.5$. Of the 284 patients, 177 (62.3\%) had an $\mathrm{EMD} /$ mesorectum ratio $\leq 0.5$. The correlation between the $\mathrm{EMD} / \mathrm{mesorectum}$ ratio and patient characteristics was shown in Table 1. An EMD/mesorectum ratio > 0.5 occurred more often in patients with T3c (65.9\%) and T3d (100\%). Patients with tumour on anterior rectal wall, positive MRF and positive EMVI also had higher proportion of $\mathrm{EMD} /$ mesorectum ratio $>0.5$.
Figure 1. Patient with $E M D /$ mesorectum ratio $\leq 0.5$. (a). The EMD was $6.24 \mathrm{~mm}$; (b) The distance of mesorectum was $16.01 \mathrm{~mm}$. The EMD/mesorectum ratio was 0.39. EMD, extra mural depth.

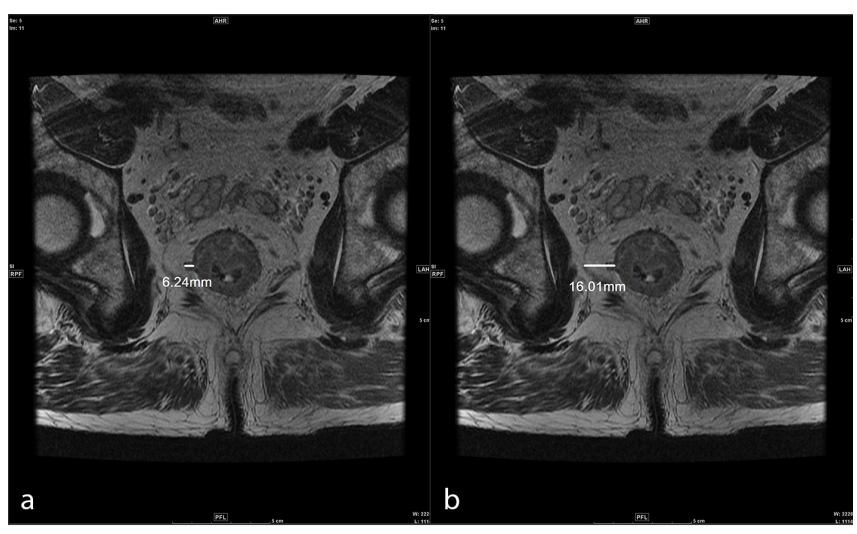

Table 2 showed the correlation between the MRI characteristics and pathological TRG score. TRG 0-1 indicates good tumour response after chemoradiation and TRG 2-3 stands for poor response. $53.2 \%$ tumour with $\mathrm{mrT3} a+\mathrm{b}$ and $53.1 \% \mathrm{EMD} /$ mesorectum ratio $\leq 0.5$ achieve TRG $0-1$ after chemoradiation with $P$ value of 0.003 and 0.006 , respectively. Patients with other characteristics including tumour in the low rectum, MRF negative and EMVI negative, also had better treatment response.

\section{Univariate and multivariate analyses}

The EMD/mesorectum ratio was significantly associated with survival. The 5-year DFS in patients with an EMD/mesorectum ratio $\leq 0.5$ was $0.794 v s 0.506$ in patients with a ratio $>0.5(p$ $=0.004$ ) (Figure 3 ). The 5 -year OS in patients with an EMD/ mesorectum ratio $\leq 0.5$ was 0.869 vs 0.705 in patients with a ratio $>0.5(p=0.047)$ (Figure 4).

In the univariate analysis of DFS (Table 3), tumour location, $\mathrm{EMD} / \mathrm{mesorectum}$ ratio, tumour direction, surgery method and ypTNM stage were associated with DFS. For OS, only patients with TRG 2-3 had decreased OS compared with patients with

Figure 2. Patient with $E M D /$ mesorectum ratio > 0.5. (a) The EMD was $10.31 \mathrm{~mm}$; (b) The distance of mesorectum was $15.70 \mathrm{~mm}$. The EMD/mesorectum ratio was 0.66. EMD, extra mural depth.

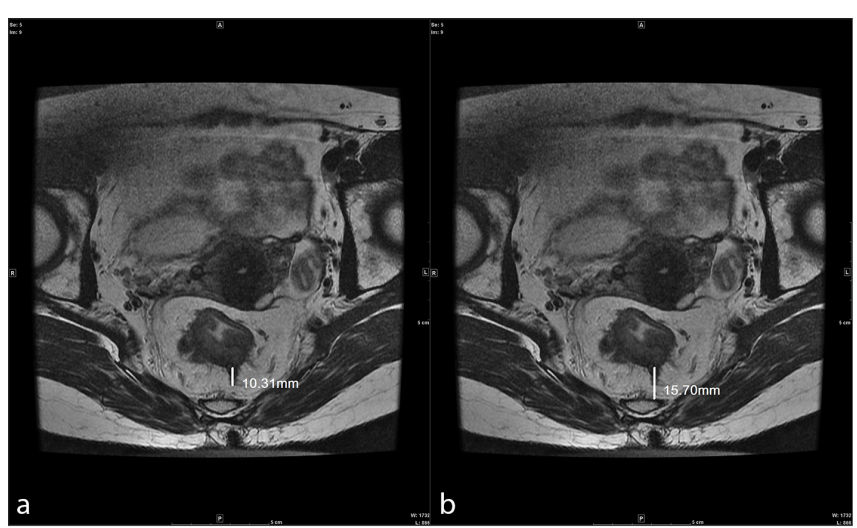


Table 1. Correlation between EMD/mesorectum ratio and patient characteristics

\begin{tabular}{|c|c|c|c|}
\hline Patient characteristics & $\mathrm{EMD} /$ mesorectum ratio $\leq 0.5, \mathrm{n}(\%)$ & $\mathrm{EMD} /$ mesorectum ratio $>0.5, \mathrm{n}(\%)$ & $p$ value \\
\hline \multicolumn{4}{|l|}{ Gender } \\
\hline Male & $129(63.5)$ & $74(36.5)$ & 0.501 \\
\hline Female & $48(59.3)$ & $33(40.7)$ & \\
\hline \multicolumn{4}{|l|}{ Tumour location } \\
\hline Low & $84(55.3)$ & $68(44.7)$ & 0.008 \\
\hline Middle-high & $93(70.5)$ & $39(29.5)$ & \\
\hline \multicolumn{4}{|l|}{ mrT stage } \\
\hline T3a & $3(100)$ & $0(0)$ & $<0.001^{a}$ \\
\hline $\mathrm{T} 3 \mathrm{~b}$ & $143(77.7)$ & $41(22.3)$ & \\
\hline $\mathrm{T} 3 \mathrm{c}$ & $31(34.1)$ & $60(65.9)$ & \\
\hline T3d & $0(0)$ & $6(100)$ & \\
\hline \multicolumn{4}{|l|}{ mrN stage } \\
\hline No & $11(55.0)$ & $9(45.0)$ & \\
\hline N1 & $81(68.1)$ & $38(31.9)$ & 0.226 \\
\hline $\mathrm{N} 2$ & $85(58.6)$ & $60(41.4)$ & \\
\hline \multicolumn{4}{|l|}{ Tumour direction } \\
\hline Anterior & $12(28.6)$ & $30(71.4)$ & \\
\hline Lateral & $136(69.7)$ & $59(30.3)$ & $<0.001$ \\
\hline Posterior & $29(61.7)$ & $18(38.3)$ & \\
\hline \multicolumn{4}{|l|}{ MRF } \\
\hline Negative & $118(79.7)$ & $30(20.3)$ & $<0.001$ \\
\hline Positive & $59(43.4)$ & 77 (56.6) & \\
\hline \multicolumn{4}{|l|}{ EMVI } \\
\hline Negative & 137 (69.5) & $60(30.5)$ & $<0.001$ \\
\hline Positive & $40(46.0)$ & $47(54.0)$ & \\
\hline \multicolumn{4}{|l|}{ ypT stage } \\
\hline 0 & $47(69.1)$ & $21(30.9)$ & $0.016^{a}$ \\
\hline 1 & $10(83.3)$ & $2(16.7)$ & \\
\hline 2 & $57(68.7)$ & $26(31.3)$ & \\
\hline 3 & $63(52.1)$ & $58(47.9)$ & \\
\hline \multicolumn{4}{|l|}{ ypN stage } \\
\hline 0 & $119(66.9)$ & $59(33.1)$ & \\
\hline 1 & $40(49.4)$ & $41(50.6)$ & 0.016 \\
\hline 2 & $18(72.0)$ & $7(28.0)$ & \\
\hline \multicolumn{4}{|l|}{ Neural invasion } \\
\hline Negative & $164(63.1)$ & $96(36.9)$ & 0.389 \\
\hline Positive & $13(54.2)$ & $11(45.8)$ & \\
\hline \multicolumn{4}{|l|}{ Vascular invasion } \\
\hline Negative & $169(62.4)$ & $102(37.6)$ & $1.000^{a}$ \\
\hline Positive & $8(61.5)$ & $5(38.5)$ & \\
\hline
\end{tabular}

EMD, extramural depth; EMVI, extramuralvascular invasion; MRF, mesorectal fascia.

${ }^{a}$ Fisher's exact test. 
Table 2. Correlation between patient characteristics and tumour response

\begin{tabular}{|c|c|c|c|}
\hline $\begin{array}{l}\text { Patient } \\
\text { characteristics }\end{array}$ & $\begin{array}{l}\text { TRG 0- } \\
1, n(\%)\end{array}$ & $\begin{array}{l}\text { TRG 2- } \\
3, n(\%)\end{array}$ & $p$ value \\
\hline \multicolumn{4}{|l|}{ Tumour location } \\
\hline Low & $83(54.6)$ & $69(45.4)$ & 0.005 \\
\hline Middle-high & $50(37.9)$ & $82(62.1)$ & \\
\hline \multicolumn{4}{|l|}{ mrT stage } \\
\hline $\mathrm{T} 3 \mathrm{a}+\mathrm{b}$ & $99(53.2)$ & $87(46.8)$ & 0.003 \\
\hline $\mathrm{T} 3 \mathrm{c}+\mathrm{d}$ & $34(34.7)$ & $64(65.3)$ & \\
\hline \multicolumn{4}{|l|}{ mrN stage } \\
\hline No & $8(40.0)$ & $12(60.0)$ & 0.306 \\
\hline N1 & $62(52.1)$ & $57(47.9)$ & \\
\hline $\mathrm{N} 2$ & $63(43.4)$ & $82(56.6)$ & \\
\hline \multicolumn{4}{|l|}{$\begin{array}{l}\mathrm{EMD} / \text { mesorectum } \\
\text { ratio }\end{array}$} \\
\hline$\leq 0.5$ & $94(53.1)$ & $83(46.9)$ & 0.006 \\
\hline$>0.5$ & $39(36.4)$ & 68 (63.6) & \\
\hline \multicolumn{4}{|l|}{ Tumour direction } \\
\hline Anterior & $18(42.9)$ & $24(57.1)$ & 0.474 \\
\hline Lateral & $96(49.2)$ & $99(50.8)$ & \\
\hline Posterior & $19(40.4)$ & $28(59.6)$ & \\
\hline \multicolumn{4}{|l|}{ MRF } \\
\hline Negative & $81(54.7)$ & $67(45.3)$ & 0.005 \\
\hline Positive & $52(38.2)$ & $84(61.8)$ & \\
\hline \multicolumn{4}{|l|}{ EMVI } \\
\hline Negative & $105(53.3)$ & $92(46.7)$ & 0.001 \\
\hline Positive & $28(32.2)$ & $59(67.8)$ & \\
\hline \multicolumn{4}{|l|}{ RT dose } \\
\hline$\leq 50$ Gy & $123(46.2)$ & $143(53.8)$ & 0.443 \\
\hline$>50 \mathrm{~Gy}$ & $10(55.6)$ & $8(44.4)$ & \\
\hline
\end{tabular}

EMD, extramural depth; EMVI, extramuralvascular invasion; MRF, mesorectal fascia; RT, radiotherapy; TRG, tumour regression grade.

TRG 0-1 [HR 2.959; 95\% CI (1.081-8.095); $p=0.035]$. Therefore, multivariate analysis was only performed for DFS.

A multivariate analysis was performed for the characteristics that had $p<0.05$ based on the univariate analysis (Table 4). Patients with an EMD/mesorectum ratio > 0.5 (HR 2.020; 95\% CI 1.078$3.786 ; p=0.028$ ) and ypTNM II-III (HR 3.550; 95\% CI 1.260$10.000 ; p=0.017$ ) had a worse DFS in the multivariate analysis.

\section{DISCUSSION}

The goal of the present work was to assess the value of the EMD/ mesorectum ratio as a potential imaging biomarker in stage $\mathrm{T} 3$ rectal cancer. The results of our study demonstrate statistically significant correlations between the $\mathrm{EMD} /$ mesorectum ratio and both chemoradiation response and survival. The frequency of
Figure 3. Disease-free survival stratified by an EMD/mesorectum ratio of 0.5. EMD, extramural depth.

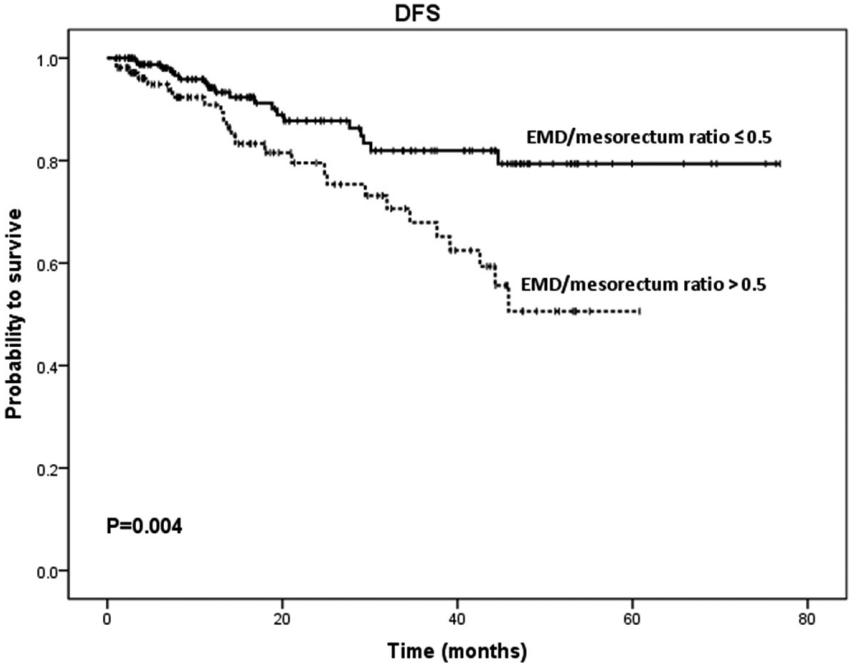

good response with TRG $0-1$ was significantly higher for patients with an EMD/mesorectum ratio $\leq 0.5(53.1 \%)$ compared with a ratio $>0.5(36.4 \%)$. Patients with an $\mathrm{EMD} /$ mesorectum ratio $\leq 0.5$ also had better DFS and trended to have better OS.

The depth of extramural penetration of the tumour has been shown to be an independent prognostic factor in $\mathrm{T} 3$ rectal cancer in some studies, and it is clear that patients with more than 5 $\mathrm{mm}$ of extramural spread should be identified because they have a markedly worse prognosis than patients with $5 \mathrm{~mm}$ or less of spread. ${ }^{6,16}$ Meanwhile, the maximal EMD obtained on the initial staging MRI can also predict the neoadjuvant chemoradiation response. ${ }^{17}$ However, the mesorectal fat layer is rather thin in Chinese patients, and the mesorectal fat thickness has been shown to be $<15 \mathrm{~mm}$ in the majority of patients in most positions and at most levels. ${ }^{11}$ Moreover, the criterion of T3a with invasion of mesorectum less than $1 \mathrm{~mm}$ is difficult to measure in the clinical practice. In our study, most patients were classified as

Figure 4. Overall survival stratified by an EMD/mesorectum ratio of 0.5. EMD, extramural depth.

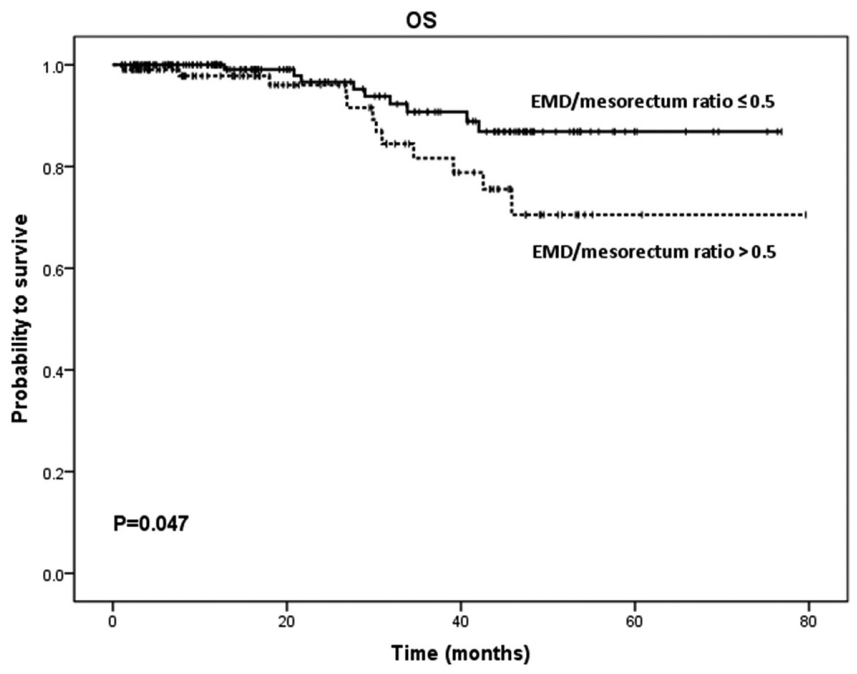


Table 3. Univariate analysis in relation to DFS and OS

\begin{tabular}{|c|c|c|c|c|}
\hline \multirow{2}{*}{$\begin{array}{l}\text { Patient } \\
\text { characteristics }\end{array}$} & \multicolumn{2}{|c|}{ DFS } & \multicolumn{2}{|c|}{ OS } \\
\hline & HR (95\% CI) & $p$ value & HR (95\% CI) & $p$ value \\
\hline \multicolumn{5}{|l|}{ Age, years } \\
\hline$\leq 60$ & $1.0(\mathrm{ref})$ & - & $1.0(\mathrm{ref})$ & - \\
\hline$>60$ & $1.265(0.689-2.322)$ & 0.449 & $1.684(0.709-4.003)$ & 0.238 \\
\hline \multicolumn{5}{|l|}{ Gender } \\
\hline Male & $1.0(\mathrm{ref})$ & - & 1.0 (ref) & - \\
\hline Female & $1.764(0.966-3.219)$ & 0.065 & $0.969(0.376-2.498)$ & 0.947 \\
\hline \multicolumn{5}{|l|}{ Tumour location } \\
\hline Low & 1.0 (ref) & - & 1.0 (ref) & - \\
\hline Middle-High & $0.435(0.224-0.845)$ & 0.014 & $0.491(0.190-1.265)$ & 0.141 \\
\hline \multicolumn{5}{|l|}{ mrT stage } \\
\hline $\mathrm{T} 3 \mathrm{a}+\mathrm{b}$ & $1.0(\mathrm{ref})$ & - & 1.0 (ref) & - \\
\hline $\mathrm{T} 3 \mathrm{c}+\mathrm{d}$ & $0.991(0.536-1.834)$ & 0.978 & $1.133(0.469-2.737)$ & 0.781 \\
\hline \multicolumn{5}{|l|}{ mrN stage } \\
\hline N0-1 & $1.0(\mathrm{ref})$ & - & 1.0 (ref) & - \\
\hline $\mathrm{N} 2$ & $1.010(0.558-1.831)$ & 0.973 & $0.743(0.308-1.794)$ & 0.509 \\
\hline \multicolumn{5}{|l|}{ EMD/mesorectum ratio } \\
\hline$\leq 0.5$ & 1.0 (ref) & - & 1.0 (ref) & - \\
\hline$>0.5$ & $2.368(1.303-4.302)$ & 0.005 & $2.343(0.987-5.564)$ & 0.054 \\
\hline \multicolumn{5}{|l|}{ Tumour direction } \\
\hline Anterior & $1.0(\mathrm{ref})$ & - & 1.0 (ref) & - \\
\hline Lateral + Posterior & $0.473(0.247-0.905)$ & 0.024 & $0.719(0.263-1.965)$ & 0.521 \\
\hline \multicolumn{5}{|l|}{ MRF } \\
\hline Negative & 1.0 (ref) & - & 1.0 (ref) & - \\
\hline Positive & $2.058(1.111-3.811)$ & 0.022 & $1.270(0.538-2.995)$ & 0.585 \\
\hline \multicolumn{5}{|l|}{ EMVI } \\
\hline Negative & 1.0 (ref) & - & 1.0 (ref) & - \\
\hline Positive & $1.592(0.876-2.892)$ & 0.127 & $1.543(0.650-3.665)$ & 0.325 \\
\hline \multicolumn{5}{|l|}{ RT dose, Gy } \\
\hline $45-50$ & $1.0(\mathrm{ref})$ & - & 1.0 (ref) & - \\
\hline$>50$ & $0.897(0.320-2.513)$ & 0.835 & $0.763(0.177-3.284)$ & 0.717 \\
\hline \multicolumn{5}{|l|}{ Surgery } \\
\hline $\mathrm{AR}$ & 1.0 (ref) & - & 1.0 (ref) & - \\
\hline APR + Hartmann & $1.974(1.045-3.729)$ & 0.036 & $1.983(0.768-5.118)$ & 0.157 \\
\hline \multicolumn{5}{|l|}{ ypTNM stage } \\
\hline $0-\mathrm{I}$ & 1.0 (ref) & - & 1.0 (ref) & - \\
\hline II-III & $3.081(1.101-8.622)$ & 0.032 & $6.019(0.807-44.872)$ & 0.080 \\
\hline \multicolumn{5}{|l|}{ TRG } \\
\hline $0-1$ & $1.0(\mathrm{ref})$ & - & $1.0(\mathrm{ref})$ & - \\
\hline $2-3$ & $1.604(0.866-2.970)$ & 0.133 & $2.959(1.081-8.095)$ & 0.035 \\
\hline
\end{tabular}

(Continued) 
Table 3. (Continued)

\begin{tabular}{|c|c|c|c|c|}
\hline \multirow{2}{*}{$\begin{array}{l}\text { Patient } \\
\text { characteristics }\end{array}$} & \multicolumn{2}{|c|}{ DFS } & \multicolumn{2}{|c|}{ OS } \\
\hline & HR (95\% CI) & $p$ value & HR (95\% CI) & $p$ value \\
\hline \multicolumn{5}{|l|}{ Nerual invasion } \\
\hline Negative & 1.0 (ref) & - & 1.0 (ref) & - \\
\hline Positive & $1.705(0.759-3.829)$ & 0.196 & $2.619(0.958-7.158)$ & 0.061 \\
\hline \multicolumn{5}{|l|}{ Vascular invasion } \\
\hline Negative & 1.0 (ref) & - & 1.0 (ref) & - \\
\hline Positive & $1.595(0.569-4.473)$ & 0.375 & $2.028(0.595-6.914)$ & 0.259 \\
\hline
\end{tabular}

DFS, disease-free survival; EMD, extramural depth; EMVI, extramural vascular invasion; HR, hazard ratio; MRF, mesorectalfascia; OS, overall survival; RT, radiotherapy; TRG, tumour regression grade; r, regression grade.

either T3b (64.8\%) and T3c (32.0\%), with only $3(1.1 \%)$ patients classified with T3a and $6(2.1 \%)$ patients with T3d. Therefore, the ratio of mesorectum invasion depth would be a good supplement for the absolute value of EMD. As a few patient numbers in T3a and T3d group, the analysis was on the combined $\mathrm{T} 3 \mathrm{a}+\mathrm{b}$ and $\mathrm{T} 3 \mathrm{c}+\mathrm{d}$. Patients with $\mathrm{T} 3 \mathrm{a}+\mathrm{b}$ achieved a higher tumour response with $53.2 \%$ TRG $0-1$ (31\%), and the group with $\mathrm{EMD} /$ mesorectum ratio $\leq 0.5$ also had similar response rate (53.1\%).

Regarding survival, the 5 -year DFS in $\mathrm{T} 3 \mathrm{a}+\mathrm{b}$ and $\mathrm{T} 3 \mathrm{c}+\mathrm{d}$ group was 67.5 and $68.7 \%$, with no significant difference among two groups (log rank $p$ value $=0.986$ ). But there was significant DFS difference in $\mathrm{EMD} /$ mesorectum ratio $\leq 0.5$ group and $>0.5$ group. Therefore, the EMD/mesorectum ratio can bring additional

Table 4. Multivariate analysis in relation to DFS

\begin{tabular}{|c|c|c|}
\hline \multirow{2}{*}{ Patient characteristics } & \multicolumn{2}{|c|}{ DFS } \\
\cline { 2 - 3 } & HR (95\% CI) & $p$ value \\
\hline Tumour location & & \\
\hline Low & $1.0(\mathrm{ref})$ & - \\
\hline Middle-high & $0.511(0.247-1.060)$ & 0.071 \\
\hline EMD/mesorectum ratio & & \\
\hline$\leq 0.5$ & $1.0(\mathrm{ref})$ & - \\
\hline$>0.5$ & $2.020(1.078-3.786)$ & 0.028 \\
\hline Tumour direction & & \\
\hline Anterior & $1.0(\mathrm{ref})$ & - \\
\hline Lateral + Posterior & $0.642(0.327-1.261)$ & 0.642 \\
\hline Surgery & & \\
\hline AR & $1.0(\mathrm{ref})$ & - \\
\hline APR + Hartmann & $1.220(0.601-2.475)$ & 0.582 \\
\hline ypTNM stage & & \\
\hline 0-I & $1.0(\mathrm{ref})$ & - \\
\hline II-III & $3.550(1.260-10.000)$ & 0.017 \\
\hline
\end{tabular}

DFS, disease-free survival; EMD, extramural depth; HR, hazard ratio. predictive and prognostic information for T3 rectal patients, especially in the Chinese population.

Since the special cone anatomy of the rectum, the low-rectum and middle-high rectum may have difference. The EMD/ mesorectum ratio is higher in the low-rectum. The ratio in the low-rectum is $0.51 \pm 0.30$ and $0.42 \pm 0.32$ in the middle-high rectum. The proportion of ratio $>0.5$ in the low-rectum is $44.7 \%$ and significantly higher than the middle-high rectum $(29.5 \%$, $p=0.008)$. A future study with a larger patient number could explore other cut-off value in different location of the rectum.

The position of the tumour can also affect the likelihood of mesorectal fat infiltration. The anterior aspect of the mesorectal fat layer is usually thinnest at all three arbitrary levels and tends to be compressed by anterior pelvic organs. ${ }^{11}$ In our study, tumours located in anterior wall had a higher percentage (71.4\%) of having an EMD/mesorectum ratio > 0.5 compared with tumours in the lateral $(30.3 \%)$ or posterior walls $(38.3 \%)$ $(p<0.001)$. Moreover, $56.6 \%$ of patients with an EMD/ mesorectum ratio $>0.5$ also had a positive MRF. More attention should be paid to patients with tumours in the anterior wall and with an EMD/mesorectum ratio > 0.5 in case of a positive MRF.

A pCR outcome is usually an indication of favourable biological tumour characteristics with fewer propensities for local or distant recurrences and an improved overall survival compared with patients with residual disease. ${ }^{18,19}$ Many recent studies are focusing on the "watch-and-wait" approach for rectal cancers with a complete clinical response (cCR) after chemoradiation therapy. ${ }^{20-22}$ This strategy is based on strict selection and follow-up with endoscopy and imaging. In addition, the question of whether adjuvant combination chemotherapy provides any benefits compared with the observed outcomes remains unanswered. ${ }^{23-25}$ Poor treatment response appears to indicate more aggressive tumour biology with worse long-term outcomes, and these patients may benefit from intensified treatment strategies. In our study, $69.1 \%$ patients with $\mathrm{EMD} /$ mesorectum ratio $\leq 0.5$ achieved ypT0 and $66.9 \%$ achieved ypN0, which was significantly higher compared with the group of ratio > 0.5.Patients with an EMD/mesorectum ratio > 0.5 also had decreased DFS. Therefore, the pretreatment imaging can select high-risk patients 
for novel intensified treatment, which limits the exposure of low-risk patients to treatment-related toxicities.

There are several limitations to this study, one of which is the retrospective analysis with a relatively short follow-up. The study is not a large-scale randomized trial and comprised data from only one centre; however, the data obtained show a strong correlation between the EMD/mesorectum ratio and tumour chemoradiation response and DFS, which warrants further study on a larger scale and long-term follow-up. Another weakness of this study is that the body mass index was not taken into account. Although a study from UK has shown that body mass index does not affect the volume of mesorectal fat, they included only a small patient cohort with 30 patients. $^{26}$

\section{CONCLUSION}

In conclusion, when the $\mathrm{EMD} /$ mesorectum ratio was applied to categorize T3 rectal cancer patients, a ratio of 0.5 can be used as a cut-off value for T3 subclassification. Patients with an $\mathrm{EMD} /$ mesorectum ratio $\leq 0.5$ had a higher treatment response and better survival outcomes. Large-scale prospective studies and a validation study are warranted to confirm the predictive and prognostic significance of the EMD/mesorectum ratio.

\section{FUNDING}

This study was supported by the grants from Imaging Foundation of Fudan University Shanghai Cancer Center (YX201601 and YX201602)

\section{ETHICAL STANDARDS}

This study was conducted in accordance with the World Medical Association Declaration of Helsinki, all its amendments, and national regulations. The study was approved by the Institutional Review Board of the Fudan University Shanghai Cancer Center (Shanghai, China) and all patients gave their informed consent before study enrollment.

\section{REFERENCES}

1. Kapiteijn E, Marijnen CA, Nagtegaal ID, Putter H, Steup WH, Wiggers T, et al. Preoperative radiotherapy combined with total mesorectal excision for resectable rectal cancer. $N$ Engl J Med 2001; 345: 638-46. doi: https://doi.org/10.1056/ NEJMoa010580

2. Sauer R, Becker H, Hohenberger W, Rodel C, Wittekind C, Fietkau R, et al. German rectal cancer study g. Preoperative versus postoperative chemoradiotherapy for rectal cancer. N Engl J Med 2004; 351: 1731-40.

3. Bosset JF, Collette L, Calais G, Mineur L, Maingon P, Radosevic-Jelic L, et al. Chemotherapy with preoperative radiotherapy in rectal cancer. $N$ Engl J Med 2006; 355: 1114-23. doi: https://doi.org/10. 1056/NEJMoa060829

4. Merkel S, Mansmann U, Siassi M, Papadopoulos T, Hohenberger W, Hermanek P. The prognostic inhomogeneity in pT3 rectal carcinomas. Int J Colorectal Dis 2001; 16: 298-304. doi: https://doi.org/10. 1007/s003840100309

5. Glynne-Jones R. Do t3 rectal cancers always need radiochemotherapy? Recent Results Cancer Res 2014; 203: 95-115. doi: https://doi.org/10.1007/978-3-319-080604_9

6. Shin R, Jeong SY, Yoo HY, Park KJ, Heo SC, Kang GH, et al. Depth of mesorectal extension has prognostic significance in patients with T3 rectal cancer. Dis Colon Rectum 2012; 55 : 1220-8. doi: https://doi.org/10.1097/DCR. ob013e31826fea6a
7. Zinicola R, Pedrazzi G, Haboubi N, Nicholls RJ. The degree of extramural spread of T3 rectal cancer: an appeal to the American joint committee on cancer. Colorectal Dis 2017; 19: 8-15. doi: https:// doi.org/10.1111/codi.13565

8. MERCURY Study Group. Extramural depth of tumor invasion at thin-section MR in patients with rectal cancer: results of the MERCURY study. Radiology 2007; 243: 132-9. doi: https://doi.org/10.1148/radiol. 2431051825

9. Battersby NJ, How P, Moran B, Stelzner S, West NP, Branagan G, et al. Prospective validation of a low rectal cancer magnetic resonance imaging staging system and development of a local recurrence risk stratification model: The MERCURY II Study. Ann Surg 2016; 263: 751-60. doi: https://doi.org/10.1097/SLA. 0000000000001193

10. Glimelius B, Tiret E, Cervantes A, Arnold D. ESMO Guidelines Working Group Rectal cancer: ESMO clinical practice guidelines for diagnosis, treatment and follow-up. Ann Oncol 2013; 24(Suppl 6): vi81-vi88. doi: https://doi.org/10.1093/annonc/mdt240

11. Wong EM, Lai BM, Fung VK, Cheung HY, Ng WT, Law AL, et al. Limitation of radiological T3 subclassification of rectal cancer due to paucity of mesorectal fat in Chinese patients. Hong Kong Med J 2014; 20: 366-70. doi: https://doi.org/10.12809/ hkmj144232

12. Edge SB, Compton CC AGF, Byrd DR, Carducci MA. American joint committee on cancer (AJCC) cancer staging manual. 7th edition. USA: Springer; 2010.

13. Ryan R, Gibbons D, Hyland JM, Treanor D, White A, Mulcahy HE, et al. Pathological response following long-course neoadjuvant chemoradiotherapy for locally advanced rectal cancer. Histopathology 2005; 47: 141-6. doi: https://doi.org/10.1111/j.1365-2559. 2005.02176.x

14. Jhaveri KS, Hosseini-Nik H, Thipphavong S, Assarzadegan N, Menezes RJ, Kennedy ED, et al. MRI detection of extramural venous invasion in rectal cancer: correlation with histopathology using elastin stain. AJR Am J Roentgenol 2016; 206: 747-55. doi: https:// doi.org/10.2214/AJR.15.15568

15. Chand M, Evans J, Swift RI, Tekkis PP, West NP, Stamp G, et al. The prognostic significance of postchemoradiotherapy high-resolution MRI and histopathology detected extramural venous invasion in rectal cancer. Ann Surg 2015; 261: 473-9. doi: https://doi.org/10.1097/SLA. 0000000000000848

16. Cho SH, Kim SH, Bae JH, Jang YJ, Kim HJ, Lee D, et al. Prognostic stratification by extramural depth of tumor invasion of primary rectal cancer based on the radiological society of North America proposal. AJR Am J Roentgenol 2014; 202: 1238-44. doi: https://doi.org/10.2214/AJR. 13.11311

17. Tong T, Sun Y, Cai S, Zhang Z, Gu Y. Extramural depth of rectal cancer tumor invasion at thin-section MRI: predicting treatment response to neoadjuvant chemoradiation. Oncotarget 2015; 6: 
30277-86. doi: https://doi.org/10.18632/ oncotarget.4623

18. Maas M, Nelemans PJ, Valentini V, Das P, Rödel C, Kuo LJ, et al. Long-term outcome in patients with a pathological complete response after chemoradiation for rectal cancer: a pooled analysis of individual patient data. Lancet Oncol 2010; 11 : 835-44. doi: https://doi.org/10.1016/S14702045(10)70172-8

19. Zorcolo L, Rosman AS, Restivo A, Pisano M, Nigri GR, Fancellu A, et al. Complete pathologic response after combined modality treatment for rectal cancer and long-term survival: a metaanalysis. Ann Surg Oncol 2012; 19: 2822-32. doi: https://doi.org/10.1245/s10434-0112209-y

20. Habr-Gama A, Gama-Rodrigues J, Sao Julião GP, Proscurshim I, Sabbagh C, Lynn PB, et al. Local recurrence after complete clinical response and watch and wait in rectal cancer after neoadjuvant chemoradiation: impact of salvage therapy on local disease control. Int J Radiat Oncol Biol Phys 2014; 88: 822-8. doi: https://doi. org/10.1016/j.ijrobp.2013.12.012

21. Bökkerink GM, de Graaf EJ, Punt CJ, Nagtegaal ID, Rütten H, Nuyttens JJ, et al. The CARTS study: chemoradiation therapy for rectal cancer in the distal rectum followed by organ-sparing transanal endoscopic microsurgery. BMC Surg 2011; 11: 34. doi: https://doi.org/10.1186/1471-2482-11-34

22. Weiser MR, Beets-Tan R, Beets G. Management of complete response after chemoradiation in rectal cancer. Surg Oncol Clin N Am 2014; 23: 113-25. doi: https://doi. org/10.1016/j.soc.2013.09.012

23. Breugom AJ, Swets M, Bosset JF, Collette L, Sainato A, Cionini L, et al. Adjuvant chemotherapy after preoperative (chemo)radiotherapy and surgery for patients with rectal cancer: a systematic review and meta-analysis of individual patient data. Lancet Oncol 2015; 16: 200-7. doi: https://doi.org/10.1016/S14702045(14)71199-4
24. Sainato A, Cernusco Luna Nunzia V, Valentini V, De Paoli A, Maurizi ER, Lupattelli M, et al. No benefit of adjuvant fluorouracil leucovorin chemotherapy after neoadjuvant chemoradiotherapy in locally advanced cancer of the rectum (LARC): long term results of a randomized trial (I-CNRRT. Radiother Oncol 2014; 113: 223-9. doi: https://doi.org/10.1016/j.radonc.2014.10.006

25. Breugom AJ, van Gijn W, Muller EW, Berglund Å, van den Broek CB, Fokstuen T, et al. Adjuvant chemotherapy for rectal cancer patients treated with preoperative (chemo)radiotherapy and total mesorectal excision: a dutch colorectal cancer group (DCCG) randomized phase III trial. Ann Oncol 2015; 26: 696-701. doi: https://doi.org/10.1093/annonc/mdu560

26. Allen SD, Gada V, Blunt DM. Variation of mesorectal volume with abdominal fat volume in patients with rectal carcinoma: assessment with MRI. Br J Radiol 2007; 80: 242-7. doi: https://doi.org/10.1259/bjr/ 66311683 